\title{
Accuracy evaluation of a new three-dimensional reproduction method of edentulous dental casts, and wax occlusion rims with jaw relation
}

\author{
Fu-Song Yuan*, Yu-Chun Sun*, Yong Wang and Pei-Jun Lü
}

The article introduces a new method for three-dimensional reproduction of edentulous dental casts, and wax occlusion rims with jaw relation by using a commercial high-speed line laser scanner and reverse engineering software and evaluates the method's accuracy in vitro. The method comprises three main steps: (i) acquisition of the three-dimensional stereolithography data of maxillary and mandibular edentulous dental casts and wax occlusion rims; (ii) acquisition of the three-dimensional stereolithography data of jaw relations; and (iii) registration of these data with the reverse engineering software and completing reconstruction. To evaluate the accuracy of this method, dental casts and wax occlusion rims of 10 edentulous patients were used. The lengths of eight lines between common anatomic landmarks were measured directly on the casts and occlusion rims by using a vernier caliper and on the three-dimensional computerized images by using the software measurement tool. The direct data were considered as the true values. The paired-samples $t$-test was used for statistical analysis. The mean differences between the direct and the computerized measurements were mostly less than $0.04 \mathrm{~mm}$ and were not significant $(\boldsymbol{P}>0.05)$. Statistical significance among 10 patients was assessed using one-way analysis of variance $(P<0.05)$. The result showed that the 10 patients were considered statistically no significant. Therefore, accurate three-dimensional reproduction of the edentulous dental casts, wax occlusion rims, and jaw relations was achieved. The proposed method enables the visualization of occlusion from different views and would help to meet the demand for the computer-aided design of removable complete dentures.

International Journal of Oral Science (2013) 5, 155-161; doi:10.1038/ijos.2013.49; published online 2 August 2013

Keywords: accuracy; computer-aided design/computer-aided manufacturing; complete denture; jaw relation; line laser scanner; reverse engineering software

\section{INTRODUCTION}

In the recent years, computer-aided design/computer-aided manufacturing $(\mathrm{CAD} / \mathrm{CAM})$ systems have increasingly been applied in dentistry. Over 20 years, many studies on the fabrication of prostheses by using CAD/CAM systems have been conducted in the field of fixed restorative dentistry. However, digital methods for removable complete denture fabrication are developing slowly, because of the complicated profile of complete dentures and the variety of materials used for their fabrication.

A complete denture is usually fabricated manually by several procedures ranging from making the preliminary impressions to denture placement; these procedures may be associated with certain problems. Namely, the traditional method is complicated, difficult and time consuming. Further, experienced prosthodontists and dental technicians are needed to fabricate good prostheses. A CAD/CAM system can streamline the fabrication procedure and resolve the associated problems. For instance, Kawahata et al. ${ }^{1}$ prepared wax complete dentures by computerized numerical control machining. In comparison with the traditional method, their approach reduced the number of procedures such as selection and arrangement of artificial teeth, flasking and resin packing. Sun et al. ${ }^{2}$ applied a specially developed CAD software to a three-dimensional (3D) integrated fabrication process including automatic arrangement of artificial teeth, semiautomatic designing of esthetic artificial gingiva and individualized base plates, and automatic construction of individualized virtual flasks according to the finished digital models of removable complete dentures. Then, rapid prototyping technology was used to finish the complete dentures with the conventional materials. Obviously, many manual procedures were eliminated, saving time and labor.

Capturing of the precise 3D shapes of edentulous dental casts, and wax occlusion rims with jaw relation is an important prerequisite for improving the CAD of complete dentures. Several methods have been used to obtain 3D images of edentulous jaws. Maeda et al. ${ }^{3}$ obtained anatomic and morphological information of edentulous residual ridges and the surrounding tissues from maxillomandibular double impressions mounted on a 3D high-speed laser scanner. Further, Lü

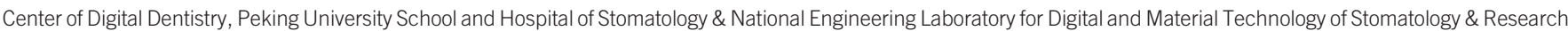
Center of Engineering and Technology for Digital Dentistry, Ministry of Health, Beijing, China

*These authors contributed equally to this study.

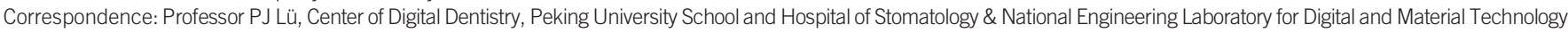
of Stomatology \& Research Center of Engineering and Technology for Digital Dentistry, Ministry of Health, 22 Zhongguancun Nandajie, Haidian District, Beijing 100081, China

E-mail: kqlpj@bjmu.edu.cn

Received 14 August 2012; accepted 26 June 2013 
et al. ${ }^{4-5}$ used a custom 3D laser scanning and measuring system to obtain the geometric parameters of edentulous jaws. Moreover, Hua and $\mathrm{Cheng}^{6}$ used a 3D coordinate-measuring machine to collect 3D information of edentulous dental casts and occlusion rims; the mathematical model of complete dentures was established by using the Bspline method.

According to the Third National Oral Health Survey, the Chinese edentulous population had reached 10 million as of 2008. Removable complete dentures are still the main prostheses used for treating edentulous patients in China. Therefore, Chinese studies of digital methods for removable complete denture fabrication are necessary. The purpose of this study was to establish a method for $3 \mathrm{D}$ reproduction of edentulous dental casts, wax occlusion rims and jaw relations by using a commercial non-contact line laser scanner and commercial reverse engineering software.

\section{MATERIALS AND METHODS}

\section{Equipment and software}

A commercial non-contact line laser scanner (D700 series; 3Shape A/S, Copenhagen, Denmark) was used. The scanner consists of a base, a laser and two high-resolution digital cameras that acquire images of the laser line as it is projected onto the model to be digitized. The three-axis motion system allows the model to be scanned from any viewpoint to ensure complete coverage of its geometry. The imageprocessing software processes the resultant images and calculates, by triangulation, a point cloud as a 3D model. The surface-creation software automatically optimizes the data and reduces the number of points in the point cloud. By combining the points into a 3D polygonal model, a 3D surface model is automatically created. To control the measurement system and process the data, a computer with an Intel Core Duo processor (no. E8400; Intel Corporation, Santa Clara, CA, USA) was used. Then, commercial reverse engineering softwareGeomagic Studio 2012(3D Systems, Morrisville, NC, USA) was used to complete the data-registration process.
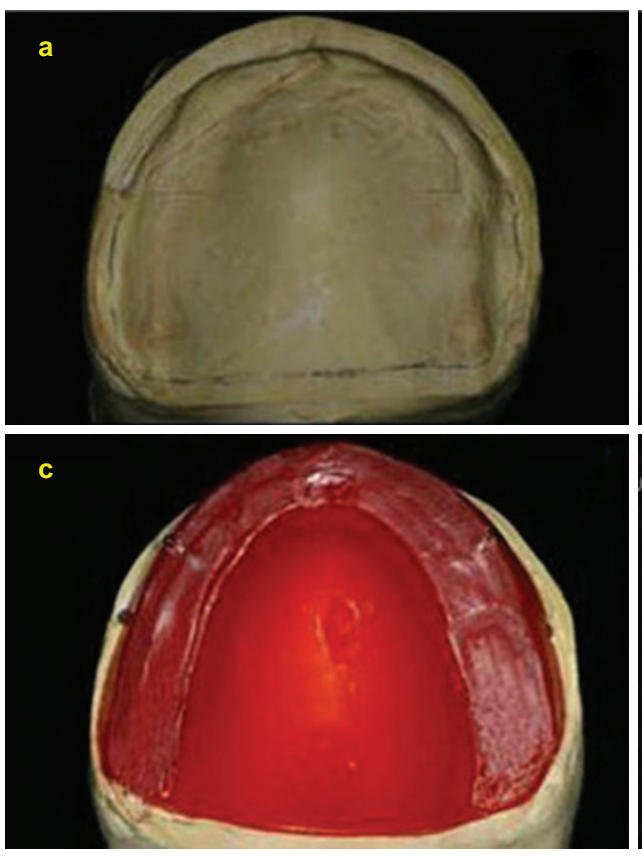

\section{Subjects}

To test the proposed method, dental casts and wax occlusion rims of 10 edentulous patients who had visited the Prosthodontics Division, Peking University Hospital of Stomatology, and had been undergoing restorative treatment to receive removable complete dentures were used. The patients had undergone extraction of all their teeth more than 3 months earlier and had well-rounded residual ridges.

\section{D reconstruction of edentulous model with jaw relation} Clinical procedures. Fabrication of dental casts and occlusion rims. First, individualized impression trays were fabricated. Then, alginate impressions were made and poured with die stone (scannable stone) to obtain edentulous dental casts. Unnecessary portions of the casts were trimmed so that blind sections could be minimized during the subsequent 3D surface-scanning process. Finally, record bases and occlusion rims were fabricated by using the conventional method ${ }^{7}$ (Figure 1).

Recording of jaw relation. To record the jaw relation, the maxillary record base was first placed into the patient's mouth and the occlusal plane was defined on the maxillary occlusion rim by using the Fox occlusal plane indicator. ${ }^{7}$ Second, the mandibular record base was placed into the patient's mouth and the vertical dimension was determined by using the rest position method. Finally, the centric relation was determined by using several direct interocclusal records and registered by using an interocclusal record material (LuxaBite; DMG Chemisch-Pharmazeutische Fabrik GmbH, Hamburg, Germany) (Figure 2).

Scanning procedures. The proposed method comprises three main steps: (i) acquisition of the 3D stereolithography (STL) data (data I) of maxillary and mandibular edentulous dental casts and wax occlusion rims; (ii) acquisition of the 3D STL data (data II) of jaw relations; and (iii) registration of data I and II with the reverse engineering
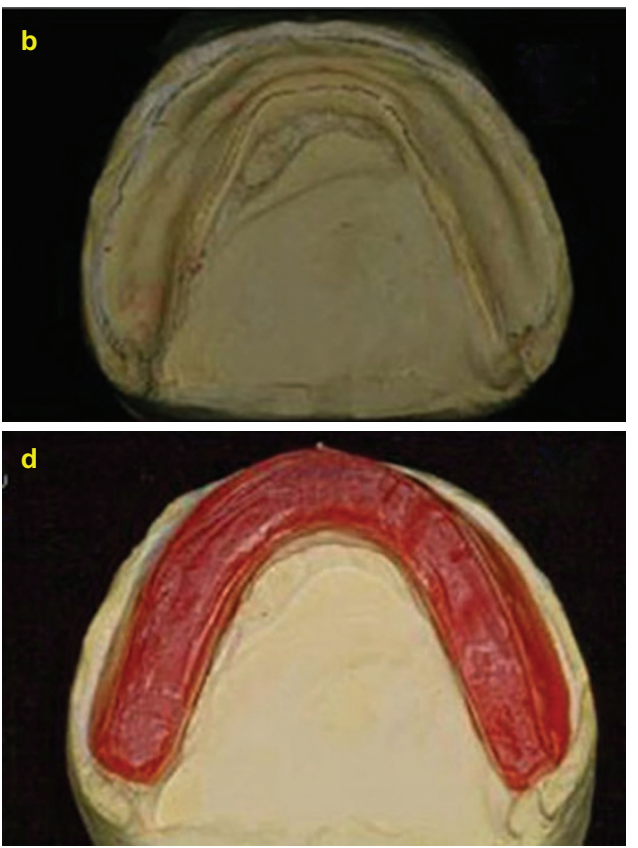

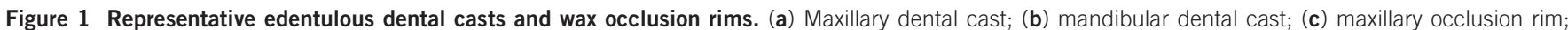
(d) mandibular occlusion rim. 


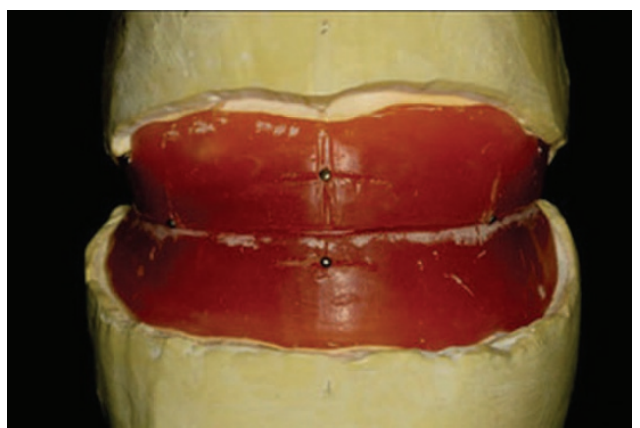

Figure 2 A representative jaw relation.

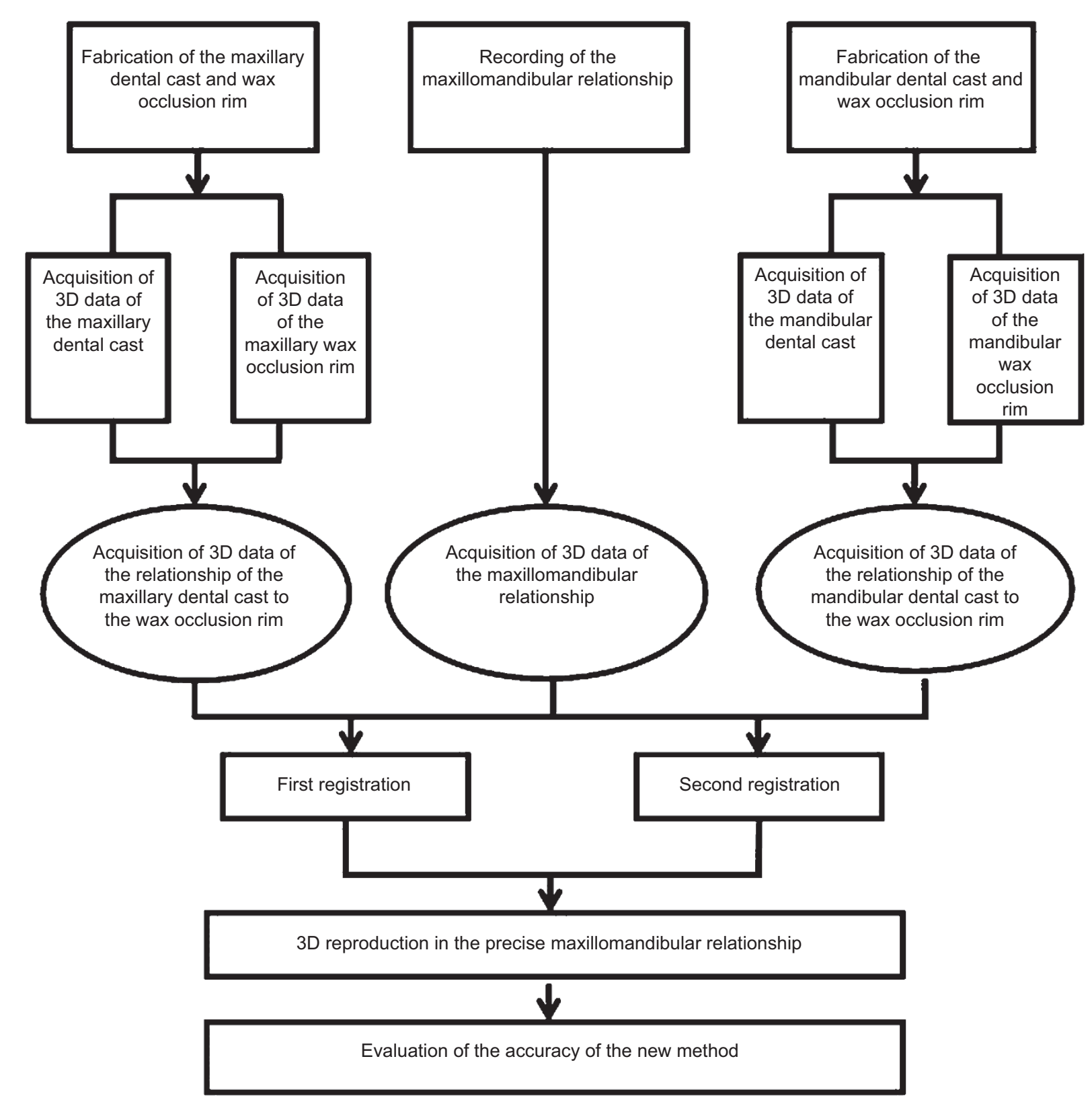

software. The process of the registration consisted of two steps. The first step was the registration of maxillary part and data II, and the second step was the registration of mandibular part and data II (Figure 3).

Step 1. Acquisition of data I. Before scanning, the maxillary and mandibular rims were covered with a thin layer of scan-spray (Bausch, Engen, Germany). By using the line laser scanner, the surfaces of the dental casts and occlusion rims were scanned, and their 3D data were acquired. Then, the relationships of the dental casts to the occlusion rims were established by the scanner's software, and these 3D data were saved in the STL format (Figure 4).

Step 2. Acquisition of data II. In this step, the line laser scanner was used to scan the surfaces of the wax occlusion rims fixed in the centric relation with the interocclusal record material, and the jaw relation

Figure 3 Overview of the 3D reproduction method. 3D, three-dimensional. 


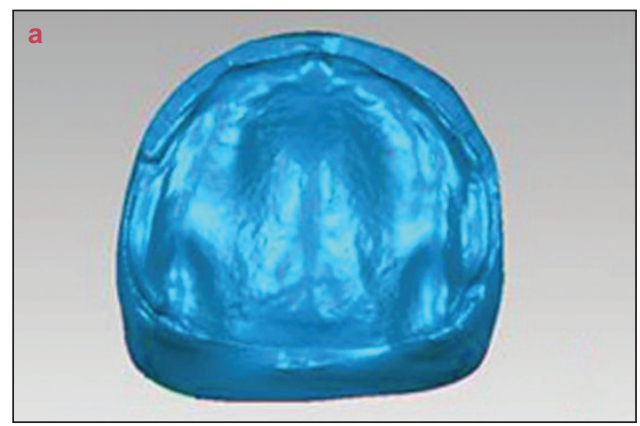

was established by the scanner's software. Again, the 3D data were saved in the STL format (Figure 5).

Step 3. Data registration and reconstruction completing. Because data II included blind sections and were incomplete whereas data I were precise and complete, they needed to be integrated and registered by using the reverse engineering software. In this way, 3D computerized images the dental casts and occlusion rims in the precise jaw relations were obtained and positioned in one coordinate system (Figures 6 and 7).
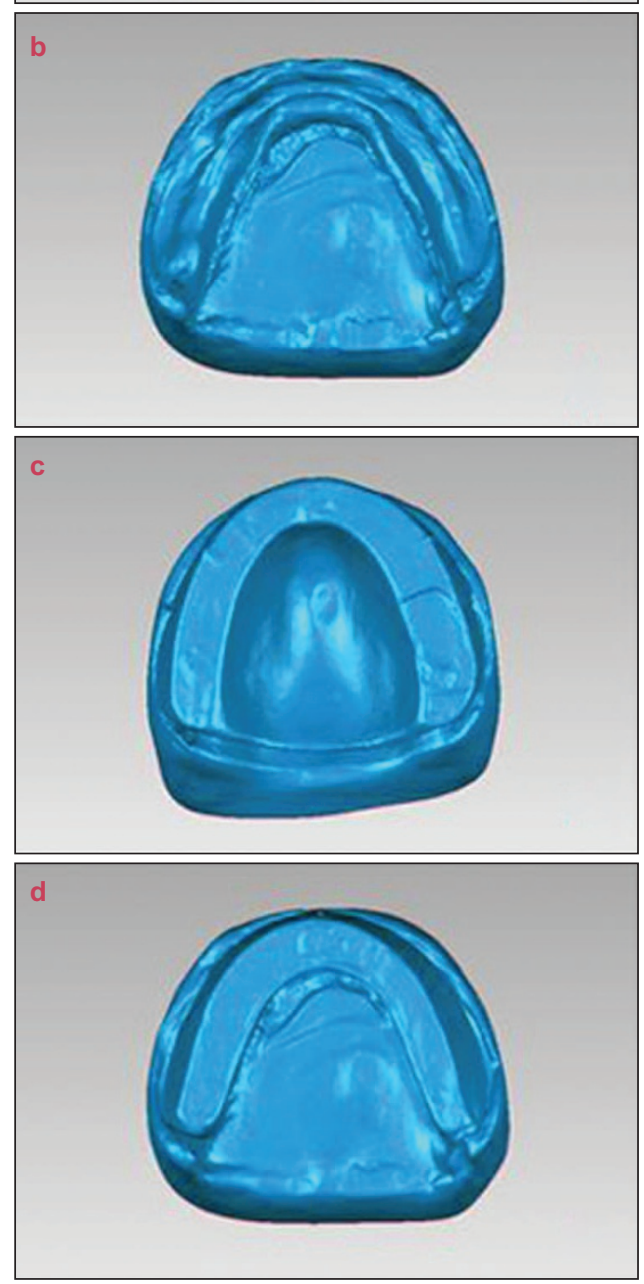

Figure 4 3D computerized images of representative edentulous dental casts and wax occlusion rims (data I). (a) Maxillary dental cast; (b) mandibular dental casts; (c) maxillary occlusion rim; (d) mandibular occlusion rim. 3D, three-dimensional.

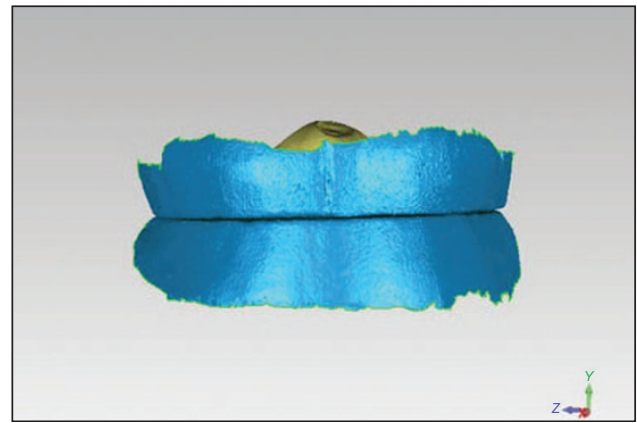

Figure 5 3D computerized image of a representative jaw relation (data II).

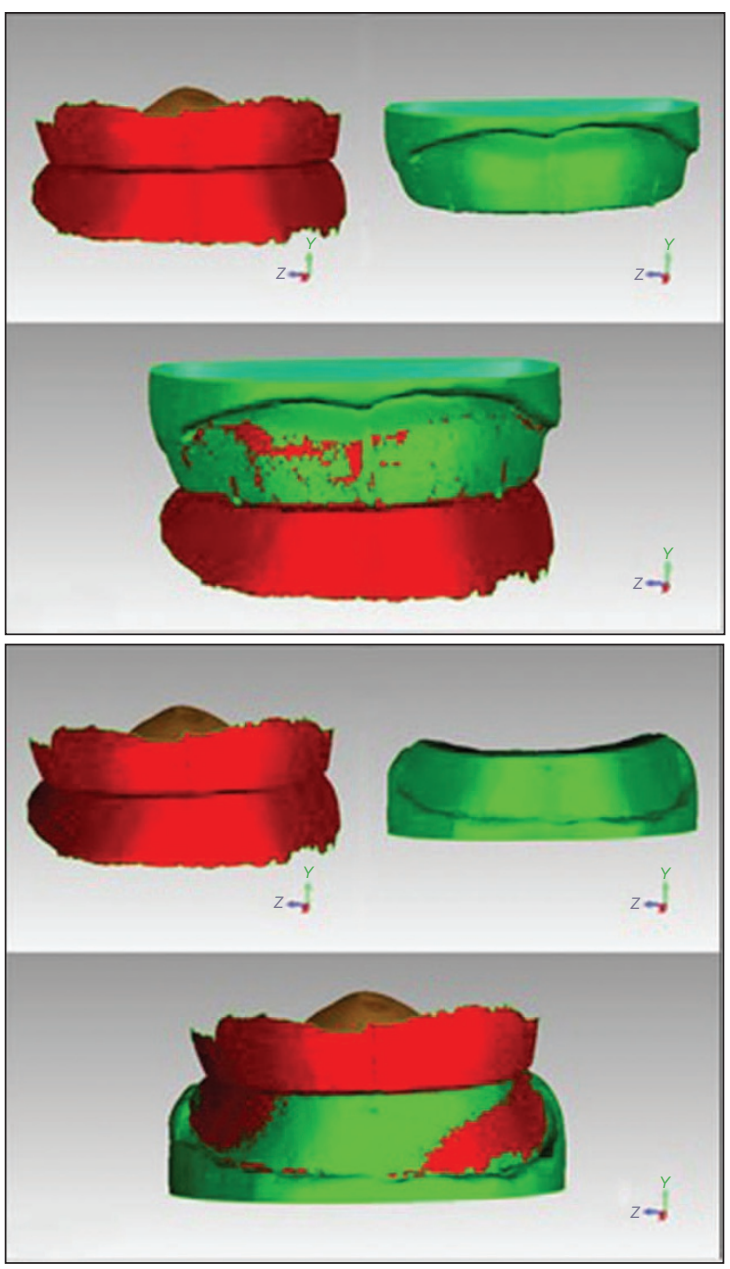

Figure 6 Process of data registration by using the reverse engineering software.

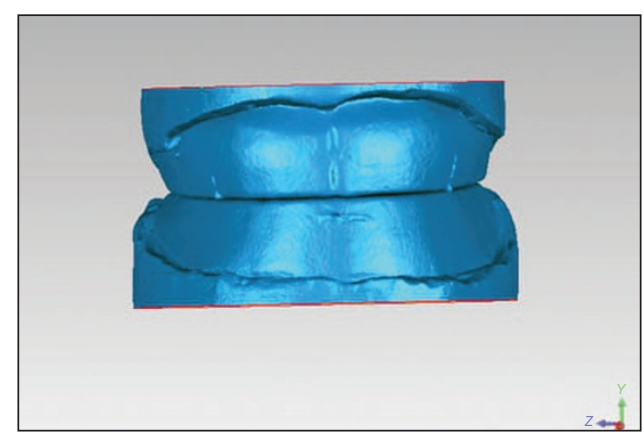

Figure 7 3D computerized image obtained after registration of data I and II. 3D, three-dimensional. 

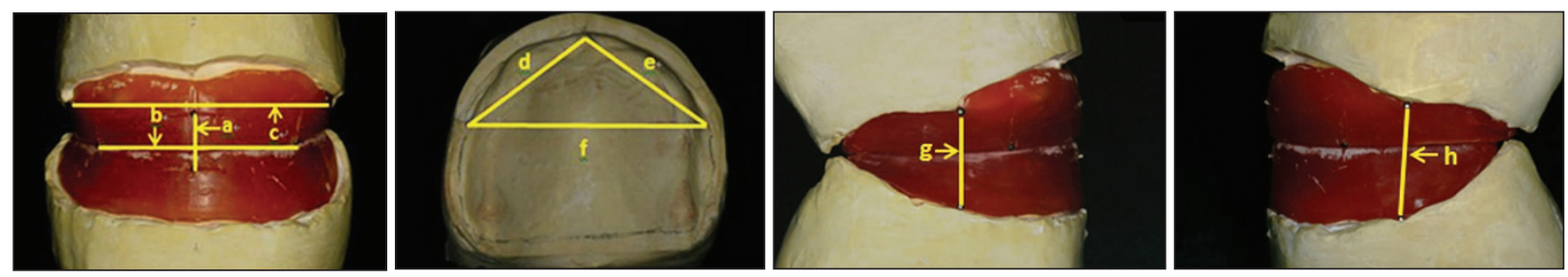

Figure 8 Measurements used for evaluating the method's accuracy. Line a: Distance between the intersections of the high and low lip lines with the midline of the occlusion rims; line $\mathrm{b}$ : distance between the markings of the corners of the mouth on the maxillary occlusion rim; line c: distance between the bilateral buccal frenal notches on the maxillary occlusion rim; line d: distance between the incisive papilla and the left buccal frenum on the maxillary dental cast; line e: distance between the incisive papilla and the right buccal frenum on the maxillary dental cast; line f: distance between the bilateral buccal frenums on the maxillary dental cast; lines $g$ and $h$ : distances between bilateral buccal frenum root points of upper jaw and ones of lower jaw.

\section{Accuracy evaluation}

To evaluate the accuracy of the proposed method, eight lines between common anatomic landmarks were first defined as shown in Figure 8. Second, their lengths were measured on the dental casts and occlusion rims and on the $3 \mathrm{D}$ computerized images by using a vernier caliper and the software measurement tool, respectively. Third, the obtained data were inputted into SPSS 19.0 software (IBM SPSS Inc., Chicago, IL, USA). The direct measurements were defined as the true values. Finally, the data of each patient were analyzed with the paired-samples $t$-test; $P<0.05$ was considered as the threshold level of significance. At the same time, statistical significance among 10 patients was assessed using one-way analysis of variance for multiple comparisons. $P<0.05$ was considered statistically significant.

\section{RESULTS}

By using the proposed method, smooth digital models of the edentulous dental casts and wax occlusion rims were obtained and the precise jaw relations were established. Information such as the anatomy of the edentulous jaws and the post-dam zone, vertical and horizontal jaw relations, position of the occlusal plane, facial midline and fullness requirements of the upper lip were converted into clear 3D data. The scanning speed was high because the acquisition of all the data was achieved in less than $20 \mathrm{~min}$.

In terms of the method's accuracy, results of measurements of eight mark lines of 10 edentulous patients by using a vernier caliper and the software measurement tool and their difference were displayed (Table 1). The mean differences between the direct and the computerized measurements were generally less than $0.04 \mathrm{~mm}$. The difference between means was not significant $(P>0.05$; Table 2$)$. The influence of various patients on accuracy of the new method was depicted in Tables 3 and 4. One-way analysis of variance revealed that 10 patients had no significant influence on accuracy of the new method $\left(F=0.714<F_{0.05}(9,70), P=0.694>0.05\right)$.

Table 1 Results of the measurements of the 10 edentulous patients

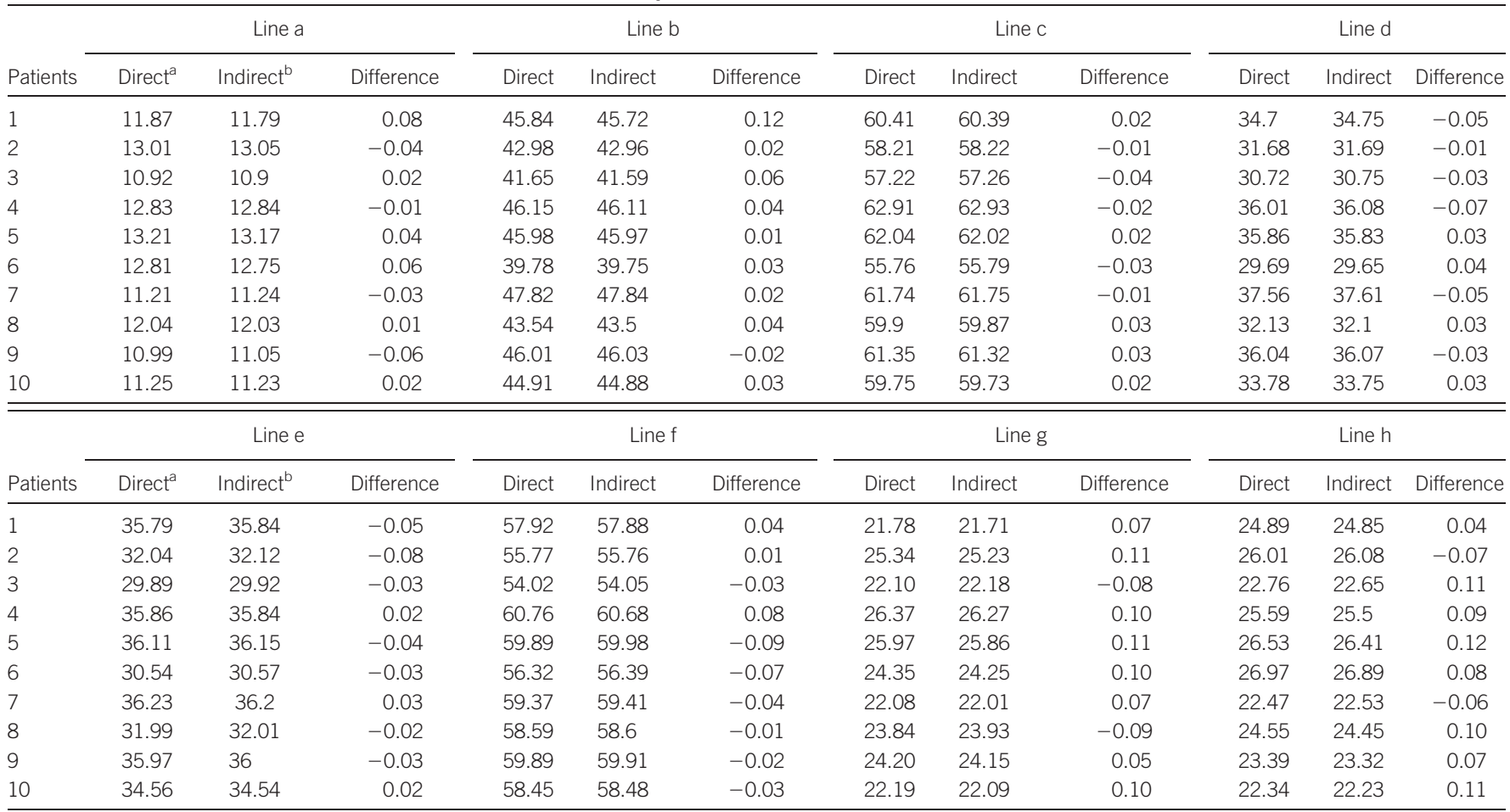

${ }^{a}$ Direct indicates the measurements obtained from the dental casts and occlusion rims by using a vernier caliper.

${ }^{b}$ Indirect indicates the measurements obtained from the 3D computerized images by using the software measurement tool. 
Table 2 Results of the paired-samples $t$-test

\begin{tabular}{lrrrr}
\hline Patients & \multicolumn{1}{c}{ Mean \pm s.d. } & \multicolumn{1}{c}{$t$} & $\mathrm{df}$ & $P$ (two-tailed) \\
\hline 1 & $0.03375 \pm 0.05999$ & 1.591 & 7 & 0.156 \\
2 & $-0.00875 \pm 0.05987$ & -0.413 & 7 & 0.692 \\
3 & $-0.00250 \pm 0.06182$ & -0.114 & 7 & 0.912 \\
4 & $0.02875 \pm 0.06010$ & 1.353 & 7 & 0.218 \\
5 & $0.02500 \pm 0.06990$ & 1.012 & 7 & 0.345 \\
6 & $0.02250 \pm 0.05994$ & 1.062 & 7 & 0.324 \\
7 & $-0.01375 \pm 0.04373$ & -0.889 & 7 & 0.403 \\
8 & $0.01125 \pm 0.05489$ & 0.580 & 7 & 0.580 \\
9 & $-0.00125 \pm 0.04549$ & -0.078 & 7 & 0.940 \\
10 & $0.03125 \pm 0.05167$ & 1.711 & 7 & 0.131 \\
\hline
\end{tabular}

s.d., standard deviation; df, degrees of freedom.

Table 3 Test of homogeneity of variances

\begin{tabular}{lccc}
\hline Levene statistic & df1 & df2 & Sig \\
\hline 0.358 & 9 & 70 & 0.351 \\
\hline
\end{tabular}

df, degrees of freedom; Sig, significance.

Table 4 One-way analysis of variance

\begin{tabular}{|c|c|c|c|c|c|}
\hline Variances & $\begin{array}{l}\text { Sum of } \\
\text { squares }\end{array}$ & $\mathrm{df}$ & $\begin{array}{c}\text { Mean } \\
\text { squares }\end{array}$ & $F$ & Sig \\
\hline Between groups & 0.021 & 9 & 0.002 & 0.714 & 0.694 \\
\hline Within groups & 0.231 & 70 & 0.003 & & \\
\hline Total & 0.252 & 79 & & & \\
\hline
\end{tabular}

df, degrees of freedom; Sig, significance; F, freedom.

\section{DISCUSSION}

The compatibility of a dental restoration depends on quality throughout the entire CAD/CAM process. Quality is affected by several factors such as the impression technique, ${ }^{8}$ production of the dental cast, acquisition of the digital model, CAD and CAM. In processes based on CAD/CAM technology, the transfer of geometric data starts with surface digitization. ${ }^{9}$

The current $3 \mathrm{D}$ scanners used in oral medicine are classified as touch-probe scanners and non-contact scanners. ${ }^{10}$ The main merits of touch-probe scanners are their inexpensiveness and efficiency for acquiring data of objects with low geometric surface detail. Their demerits are the time-consuming and impractical procedures needed for artifact digitization. ${ }^{11}$ Non-contact scanning includes laser scanning and photogrammetry. The basis of this technology is laser triangulation. Laser scanning is not only the most established but also the most widely used method. In laser scanning, the laser is emitted onto the model and the reflected patterns are registered by a digital camera. After the reflections are tracked as images, a 3D point cloud is obtained by using triangulation technology. In general, laser scanning allows the scanning of soft and brittle materials. Laser scanning methods include point and line laser scanning. The point laser scanner has a long scanning time because the laser must travel across every point on the model. ${ }^{12}$ In comparison, the line laser scanner affords several advantages such as high scanning speed, high resolution, acquisition of large volumes of data, great working distance and high scanning precision for fine anatomic structures.

Because of these advantages, line laser scanners are widely applied in dentistry. For example, in 1992, Soma et al. ${ }^{13}$ developed a line laser scanner that utilizes a flat laser beam to produce accurate measurements and enable the simultaneous measurement of 200 points on a 30 -mm-long line; it requires $8-10 \mathrm{~min}$ to digitize the whole morphology of the model. In 1996, Kuroda et al. ${ }^{14}$ developed a 3D dental castanalyzing system with laser scanning. The $3 \mathrm{D}$ graphics of the model can be generated in approximately $40 \mathrm{~min}$, and the measurement error is less than $0.05 \mathrm{~mm}$. In 1997, Mehl et al. ${ }^{15}$ used a line laser scanner to detect the wear of the functional surfaces of restorations. In 2011, Chen et al. ${ }^{16}$ obtained geometric models of tooth preparations and full crowns by using a line laser scanner and established a digital method for evaluating the absolute marginal discrepancy of full crowns. Further, Liu et al. ${ }^{17}$ used a line laser scanner to acquire the $3 \mathrm{D}$ data of prepared teeth, neighboring teeth and antagonist teeth and obtained quantitative parameters for evaluating tooth preparations to receive artificial crowns. However, according to the literature, line laser scanning is mainly applied in fixed prosthodontics and seldom to removal denture fabrication.

In this study, the data-registration process could increase the mean error. The Iterative Closest Point algorithm is applied mainly in this process and should therefore be improved to ensure precise data registration. The time required for the entire $3 \mathrm{D}$ reproduction was about $20 \mathrm{~min}$. It can be shortened if an exclusive measurement program is developed, the Iterative Closest Point algorithm is improved or a computer with higher performance is used. However, the proposed method is an effective solution to replace stone casts with $3 \mathrm{D}$ digital information. This method is assumed to meet the submillimetric accuracy requirements for the $\mathrm{CAD}$ of complete dentures and could pave the way for the CAD of complete dentures.

The method can also be used to obtain the 3D data of jaw relations, which are one of the most important elements in complete denture fabrication, because their accuracy may determine the accuracy of the complete dentures. Jaw relations of complete dentures are closely related to mucosal characteristics. For example, according to $\mathrm{Hu}$ et al. ${ }^{18}$ the least thickness of the oral mucosa of edentulous patients is $1.78-1.94 \mathrm{~mm}$. When a load of about $0.2 \mathrm{~g} / \mathrm{mm}^{2}$ is applied because of the use of complete dentures, the thickness can be reduced by $20 \%$. Therefore, the deformation level of the oral mucosa is about $350 \mu \mathrm{m}$. Zhong et al. ${ }^{19}$ measured loads at different points and the corresponding amounts of deformation of the oral mucosa when making edentulous impressions by using the 3D finite element method and concluded that the maximum amount of deformation is $314 \mu \mathrm{m}$, corresponding to a load of $56.43 \mathrm{~g} \cdot \mathrm{mm}^{-2}$. The reproduction accuracy of jaw relations is about $150 \mu \mathrm{m}$, which is half of the maximum amount of deformation of the oral mucosa. Therefore, the accuracy of this method may fulfill the requirements for removable complete denture fabrication.

A limitation of this method is that the $3 \mathrm{D}$ data of a single wax occlusion rim cannot be acquired; the 3D data of dental casts and occlusion rims are acquired together. Therefore, the repeated data could reduce the speed and accuracy of the registration. In future studies, a method for obtaining the $3 \mathrm{D}$ data of single wax occlusion rims will be investigated.

\section{CONCLUSIONS}

In this study, a novel method for 3D reproduction of edentulous dental casts, wax occlusion rims and jaw relations was attempted by using a commercial non-contact line laser scanner and commercial reverse engineering software. By this method, smooth $3 \mathrm{D}$ shapes of maxillary and mandibular dental casts and occlusion rims can be reproduced and jaw relations can be recorded precisely. This method 
enables the visualization of occlusion from different views and would help to meet the demand for the CAD of removable complete dentures.

\section{ACKNOWLEDGEMENTS}

This study was supported by the Twelfth Five-Year National Key Technologies Research and Development Program of China (grant no. 2012BAI07B00), the National High Technology Research and Development Program ('863' Program) of China (grant nos. 2013AA040801 and 2013AA040802), the National Natural Science Foundation of China (grant no. 81271181) and the Scientific Research Innovation Foundation for Youth Doctors of Peking University School of Stomatology (2011). The authors thank the Department of Prosthodontics, Peking University School and Hospital of Stomatology, for the study models.

1 Kawahata N, Ono H, Nishi Y et al. Trial of duplication procedure for complete dentures by CAD/CAM. J Oral Rehabil 1997; 24(7): 540-548.

2 Sun YC, Lü PJ, Wang Y. Study on CAD\&RP for removable complete denture. Comput Methods Programs Biomed 2009; 93(3): 266-272.

3 Maeda Y, Minoara M, Tsutsumi S et al. A CAD/CAM system for removable denture. Part I: fabrication of complete denture. Int J Prosthet 1994; 7(1): 17.

4 Lü PJ, Wang Y, Li GZ et al. Development of a system for robot aided teeth alignment of complete denture. Chin J Stomatol 2001; 36(2): 139-142.

5 Lü PJ, Li ZK, Wang Y et al. A study of dental cast by using 3D-laser non-contact measurement and analysis. Chin J Stomatol 1999; 34(6): 351-353.

6 Hua XM, Cheng XR. Development and applications of a computer aided complete denture design system. Hua Xi Kou Qiang Yi Xue Za Zhi 2001 19(4): 235-236.

7 Feng HL, Xu J. Prosthodontics. Beijing: Peking University Medical Press, 2005
8 Luthardt RG, Koch R, Rudolph $\mathrm{H}$ etal. Qualitative computer aided evaluation of dental impressions in vivo. Dent Mater 2006; 22(1): 66-76.

9 Luthardt R, Weber A, Rudolph $\mathrm{H}$ et al. Design and production of dental prosthetic restorations: basic research on dental CAD/CAM technology. Int J Comput Dent 2002; 5(2/3): 165-176.

$10 \mathrm{Han} Q$, Zhang FQ. Three-dimensional measurement technique applied to stomatology. Chin J Dent Mater Dev 2003; 12(1): 39-41.

11 Sinescu C, Negrutiu M, Faur N et al. Dental scanning In CAD/CAM technologies: laser beams. Int Soc Opt Eng 2008; 6843: 68430E1-68430E9.

12 Yamamoto K, Toshimitsu A, Mikami T et al. Optical measurement of dental cast profile and application to analysis of 3D tooth movement in orthodontics. Front Med Biol Eng 1988; 1(2): 119-130.

13 Soma K, Hisano M, Kuroki T et al. High accuracy measuring device for dental castusing device with flat laser beam. Kokubyo Gakkai Zasshi 1992; 5(1): 259-264.

14 Kuroda T, Motohashi N, Tominaga R et al. 3D dental cast analyzing system using laser scanning. Am J Orthod Dentofacial Orthop 1996; 110(4): 365-369.

15 Mehl A, Gloger W, Kunzelmann KH et al. A new optical 3D device for the detection of wear. J Dent Res 1997; 76(11): 1799-1807.

16 Chen YG, Lü PJ, Wang Y et al. Evaluating the absolute marginal discrepancy of full crown with a digital method. Stomatology 2011; 31(6): 321-323.

17 Liu SX, Lü PJ, Wang Y et al. Computer-assisted methods for obtaining quantitative parameters of tooth preparation for crown. Beijing J Stomatol 2011; 19(4): 190-193.

18 Hu YP, Yu SY. Characteristics of edentulous jaws. Foreign Med Sci 1933; 20(3): 159 162.

19 Zhong Q, Shen QY, Wu XY et al. The stress distribution of mandibular alveolar mucosa under functional complete denture studied by 3-D finite element analysis. Shanghai J Stomatol 2012; 21(1): 31-35.

This work is licensed under a Creative Commons Attribution-NonCommercial-NoDerivative Works 3.0 Unported License. To view a copy of this license, visit http:// creativecommons.org/licenses/by-nc-nd/3.0 